\title{
Frontières
}

\section{La fin de Don Giovanni}

\section{L'entrée en scène du libertin chez Mozart et Stravinsky}

\section{Nicoletta Diasio}

Volume 20, numéro 2, printemps 2008

Les musiques et la mort

URI : https://id.erudit.org/iderudit/018331ar

DOI : https://doi.org/10.7202/018331ar

Aller au sommaire du numéro

\section{Éditeur(s)}

Université du Québec à Montréal

ISSN

1180-3479 (imprimé)

1916-0976 (numérique)

Découvrir la revue

Citer cet article

Diasio, N. (2008). La fin de Don Giovanni : l'entrée en scène du libertin chez Mozart et Stravinsky. Frontières, 20(2), 32-36. https://doi.org/10.7202/018331ar
Résumé de l'article

Par l'analyse de deux « entrées en scène » du libertin, celle du Don Giovanni de Mozart et Da Ponte (1787) et celle de The Rake's Progress de Stravinsky et Auden (1951), l'article pointe les transformations du rapport à la mort et aux morts en Europe. Ces deux opéras représentent aussi l'apogée et le déclin du mythe de Dom Juan et de cette connexion entre le thème de l'inconstance amoureuse et celui de l'offense et de l'invitation au mort. d'utilisation que vous pouvez consulter en ligne.

https://apropos.erudit.org/fr/usagers/politique-dutilisation/ 
Résumé

Par l'analyse de deux "entrées en scène » du libertin, celle du Don Giovanni de Mozart et Da Ponte (1787) et celle de The Rake's Progress de Stravinsky et Auden (1951), l'article pointe les transformations du rapport à la mort et aux morts en Europe. Ces deux opéras représentent aussi l'apogée et le déclin du mythe de Dom Juan et de cette connexion entre le thème de l'inconstance amoureuse et celui de l'offense et de l'invitation au mort.

Mots clés: anthropologie - Europe libertin-mort-musique.

\begin{abstract}
In analysing two "stage entrance" occurences of the libertine, Mozart and Da Ponte's Don Giovanni (1787) and Stravinsky and Auden's The Rake's Progress (1951), the article highlights transformations in the relationship towards death and the dead in Europe. Both operas also represent the rise and fall of the myth of Dom Juan and of this connection between the themes of the inconstancy of love and that of the insult and invitation to the dead.
\end{abstract}

Keywords: anthropology - death Europe - libertine - music.

$\begin{array}{llllll}R & \mathrm{~T} & \mathrm{C} & \mathrm{L} & \mathrm{E} & \mathrm{S}\end{array}$

\section{LA FIN DE DON GIOVANNI}

\section{L'entrée en scène du libertin chez Mozart et Stravinsky}

\section{Nicoletta Diasio, \\ maître de conférences, Université Marc-Bloch, CNRS UMR 7043, \\ Cultures et sociétés en Europe, Strasbourg.}

L'histoire de Don Juan croise deux motifs narratifs, celui du séducteur et celui de l'offense et de l'invitation au mort, qui s'inscrivent à la fois dans le débat théologique du XVII ${ }^{\mathrm{e}}$ siècle et dans des folktales très répandues en Europe sur les dangers qui menacent ceux qui, se dérobant aux devoirs de la pietas envers les défunts, se soustraient aussi au respect des normes divines (Gendarme de Bevotte, 1911; Farinelli, 1946; Rousset, 1978; Niola, 2003). L'incontinence sexuelle du libertin est inséparable d'une indiscipline ontologique qui confond morts et vivants, nourriture mortelle et céleste, présent et passé. Ce substrat populaire, qu'on retrouve encore aujourd'hui dans le culte des âmes du Purgatoire à Naples ${ }^{1}$, a vivifié un personnage à la fortune incommensurable dans l'histoire de la littérature, des arts et de la musique. Le nombre des variations du mythe de Don Juan rivalise avec celui des écrits critiques qui lui ont été consacrés : le vertige du chiffre mille e tre, cette faim insatiable de conquête et de vie, contamine ceux qui s'approchent du personnage : mille et trois femmes (rien qu'en Espagne!), mille et trois séducteurs... Chaque lecture du Don Juan participe ainsi à en renouveler le mythe, au point que parfois l'œuvre et son interprétation se confondent, donnant lieu à une nouvelle variante : il en a été ainsi pour Hoffmann, Kierkegaard, Camus, Jouve, Macchia pour n'en citer que quelques-unes. Car, enfant d'une société contre-réformiste traversée par le frisson de la mort, des intentions moralisatrices d'un théologien jésuite, Paolo Zehentner, et d'un moine espagnol, Tirso de Molina, Don Juan incarne l'émergence d'une modernité empreinte de la conscience de la fin et de l'instabilité, où : «le monde n'est qu'une branloire pérenne. Toute chose y branle sans cesse : la terre; les rochers du Caucase, les pyramides d'Égypte, et du branle public et du leur. La constance même n'est autre chose qu'un branle plus languissant» (Montaigne, 1965, p. 44). Les variations du mythe de Don Juan nous parlent ainsi de la manière dont la société occidentale a progressivement modifié son rapport à la mort et aux morts. L'analyse de deux "entrées en scène» du séducteur, celle du Don Giovanni de Mozart et Da Ponte (1787, discographie) et celle de The Rake's Progress de Stravinsky et Auden (1951, discographie), vont me permettre d'aborder cette question par le recours à deux opéras exemplaires de l'apogée et du déclin du mythe.

\section{UN HOMME EN CAVALE}

Quand Mozart et Da Ponte, son librettiste, écrivent Don Giovanni, ce séducteur enchantait le théâtre et la littérature depuis au moins un siècle et demi: on jouait ses aventures en italien, espagnol, français, anglais, vénitien, napolitain; sur des scènes aussi diverses que des salles royales ou des séminaires, des théâtres populaires ou des foires; et dans des registres et des genres tout aussi variés: la pièce pédagogique, la pantomime, les lazzi de la commedia dell'arte, le ballet baroque, le théâtre écrit, l'opera buffa, l'opéra sérieux. 
À la fin du XVIII ${ }^{\mathrm{e}}$ siècle, Don Giovanni, confondu avec la littérature libertine, semblait avoir achevé son cycle. Mais il ne mourut pas. L'affranchir de ce monde sombre et glacé, le soustraire aux gestes grossiers des comédiens qui en faisaient un pantin dont la fin n'était plus crédible, était la seule manière pour le rendre encore vivant et actuel. Et ainsi, pris par l'ardeur de représenter avec une intensité majeure le vieux personnage, Mozart en créa un totalement nouveau (Macchia, 1978, p. 40).

Don Giovanni est resté un personnage inaccompli jusqu'à la création mozartienne: si le théâtre s'est approprié ce virtuose de la parole et du travestissement, ni Tirso, ni Molière, ni Goldoni en ont fait leur chef-d'œuvre; les scénarios de la commedia dell'arte donnent souvent le premier rôle aux prouesses scéniques et acrobatiques du serviteur et aux artifices fantasmagoriques de la statue qui s'anime ou des flammes de l'enfer, selon la poétique baroque de l'émerveillement. Le chef-d'œuvre de Mozart couronne les innombrables variations du mythe de Don Juan et lui redonne un nouveau souffle. Après Mozart, Don Giovanni incarnera l'objet absolu de la musique: la mobilité, la fugacité de l'instant qui passe, ce battement du cœur et du corps qui est au fond de la vibration musicale. Mais le «sensueldémoniaque » de la musique (Kierkegaard, 1978, vol. 1, p. 118) ne va pas sans une interrogation sur la mort, sans un défi à la mort.

L'étreinte de la mort et de l'amour domine dès l'ouverture qui débute sur ce qu'Abert (1984 [1955]) interprète comme un rappel du ciel et de la transcendance, elle se poursuit ensuite dans l'enlacement entre l'Andante en ré mineur empreint d'une angoisse funéraire et le Molto allegro en ré majeur flamboyant de vie. La première scène du drame donne corps et mouvement à ce double thème. Il fait nuit, comme dans la plupart des épisodes de la carrière donjuanesque, et nous sommes dans le jardin de la demeure du Commandeur. Leporello, le serviteur, fait les cent pas devant la demeure de Donn'Anna, où son maître est en train de faire "le galant homme». Suivant la convention du mélodrame, Mozart introduit le personnage principal par le regard d'un personnage secondaire, même si Leporello est loin d'être uniquement le domestique du libertin, il en est la mémoire, le double, la conscience parfois (Rank, 1973). Tous les autres personnages entrent en contact avec Don Giovanni par la médiation du serviteur et ce dernier, toujours en scène, est aussi un interlocuteur du public, en direction de qui il com- mente les exploits du maître, parfois avec une jalousie mal cachée. Son monde est celui du quotidien, des relais, des auberges. C'est aussi le monde du sens commun et du temps «météorologique», de la pluie et du vent, des envers de l'aventure : les repas insuffisants et les nuits sans sommeil. L'air est chanté sur un ton de mauvaise humeur et de revanche impuissante: un morceau en trois parties sur un rythme de marche militaire, où les battement des tambours sont remplacés par les fouettés ascendants des instruments à cordes, mais il s'agit d'une marche qui rappelle presque les garnisons d'opérette: la «sentinelle» dont il est question attend un noble qui amourache, le gentilhomme n'a plus rien de l'idéal chevaleresque et les répétitions du «non» sur des staccati accentuent le ton de reproche contrarié de Leporello. L'obstination du valet s'anime progressivement d'une certaine agitation de l'orchestre: du monde arrive, la sentinelle se cache.

Sur ce mouvement musical qui dérobe le serviteur au regard des nouveaux arrivants, Don Giovanni et Donn'Anna font littéralement irruption sur scène. Da Ponte reprend ici l'intuition de Bertani et Gazzaniga qui, dans l'opéra Il convitato di pietra de 1787, avaient anticipé l'homicide du Commandeur dès la première scène. Cette anticipation de grand effet dramatique nous donne d'emblée le caractère fondamental du séducteur: Don Giovanni est un homme en cavale. Poursuivi, traqué dès son apparition, nous l'imaginons se déplaçant d'un bout à l'autre du grand théâtre du monde avec la désinvolture de qui a fait de la fuite le fondement de sa vie.

Ce thème est au cœur de toutes les variantes de l'intrigue. "Selle les chevaux, Catalinon! », est l'ordre qui rebondit d'une page à l'autre du drame de Tirso. Toute une topographie fantastique s'offre aux yeux du lecteur ou du spectateur: de Naples aux côtes de la Sicile, de l'Espagne à l'Orient le plus imaginaire, des mers du Nord grises et glacées à Prague, blessure sanglante au cœur de l'Europe. Tempêtes et naufrages, poursuites à cheval et vagabondages vertigineux ne pouvaient pas séduire un auteur amoureux de la mesure et des petites joies du quotidien comme Goldoni qui, critiquant l'invraisemblance des intrigues donjuanesques, épure son Don Giovanni Tenorio «de toutes les folies et les impropriétés»: le résultat en sera bien médiocre. C'est une constante qui accompagnera aussi les variantes romantiques du mythe: chez De Musset, Don Juan est "un océan devenu terre ferme», Byron en fait un héros picaresque, Puškin en fait un amant nostalgique déchiré entre la grisaille parisienne et la douceur des nuits parfumées de lauriers et de citrons.
L'empio punito, le libertin est un homme sans limites: aux espaces ouverts, changeants, souvent fantastiques explorés par notre héros s'opposent toute une série de lieux délimités, intensément socialisés, claustrophobes parfois: couvents, demeures, cours, jardins fermés et ruelles sombres, fenêtres entrouvertes et jalousies fermées. Don Giovanni est l'homme sans limites, qui entre là où il ne devrait pas. Mais il est aussi l'homme à la limite par qui se confrontent le pouvoir rassurant de l'institution et de la forme et celui dangereux de la marginalité et de l'informel. Le scandale $d u$ "profanateur» est dans ce chevauchement de plans. Il existe à ce propos toute une géographie de la marge: la rive de la plage où il échoue après un naufrage (Tirso de Molina, Byron, de Musset), le bord de la forêt (dans toutes les versions du XVII ${ }^{\mathrm{e}}$ siècle), les remparts de la ville (Puškin, Lenau), les murs du couvent (Dumas, Zorrilla) et encore les fenêtres et les portes de palais, jardins, monastères qui s'ouvrent et se referment avec circonspection, tout renvoie à une mise en scène de la marge. La frontière ultime est le mur d'enceinte du cimetière et le tombeau du Commandeur au cœur de cette succession de seuils. La position liminaire de Don Giovanni en fait le médiateur d'un intense dialogue entre les vivants et les morts, entre les hommes et les femmes, entre les espaces sacrés et profanes. Elle explique aussi la distribution des airs chez Mozart: privé d'un véritable air de caractère, le libertin est le produit des regards de tous les personnages du drame. Comme le faisait remarquer Kierkegaard, tous parlent de lui, mais il ne parle jamais de soi. Les seuls airs de Don Giovanni, par exemple l'aria dello champagne sont plutôt des relances à l'action que des moments d'effusion sentimentale ou psychologique.

Mozart et Da Ponte reprennent donc le rythme d'un scénario "qui est un voyage, un dialogue, un contraste entre un vivant (ou deux vivants) et des morts [...] une farce qui, sans explication, à la fin tourne à la tragédie, avec un héros pris à la gorge, à bout de souffle, avec l'angoisse et la libération qui est spécifique aux courses vers la mort» (Garboli, 1983). La musique nous suggère cette fugue, le ton d'opera buffa vire rapidement au sérieux et au drame, la phrase mélodique monte suivant un rythme haletant pour ensuite précipiter, "droite comme une épée» (Mila, 1988, p. 61). Les deux voix sont enlacées l'une à l'autre, tout comme Donn'Annna qui tient Don Giovanni par le bras, elles se poursuivent et s'emmêlent même là où le dialogue se fragmente dans des cris et des invectives (Gente!... Servi! Al traditore!... / Taci e trema al mio furorel 
Scellerato/ Sconsigliata). Avec le geste (l'homme qui fuit, la femme qui le retient ${ }^{2}$ ) et la musique (le canon à deux voix, puis trois avec Leporello), le livret vient souligner la dimension dynamique par une dissémination d'éléments qui confèrent mobilité et rapidité à l'action : la fréquence des liquides en « 1 » et surtout en « $r$ », les choix lexicaux (précipiter, poursuivre), des images comme celle de la furie désespérée, le temps des verbes, au présent pour Don Giovanni qui ne vit que l'instant, au futur pour Donn'Anna et Leporello: Don Giovanni: Questa furia disperata Mi vuol far precipitar / Donn'Anna: Come furia disperata Ti saprò perseguitar/ Leporello: Sta' a veder che il malandrino Mi farà precipitar.

L'espace profané, le temps de la fugue, l'abandon et la déception de la femme, la course du libertin, la crainte du serviteur constituent un imbroglio d'affects mobiles qui trouvent leur correspondant musical dans des tons «éclairés par une lumière changeante et en permanence déplacée» (Mila, 1988, p. 61).

\section{LE QUESTIONNEMENT SUR LA MORT}

La fuite du séducteur est interrompue par l'arrivée du Commandeur, Donn'Anna disparaît, le canon à trois voix est remplacé par un terzetto de basses où l'on peut reconnaître d'un point de vue anthropologique la présence d'une sphère mondaine incarnée par Leporello, l'annonce d'une dimension transcendante personnifiée par le Père et cette figure liminaire, Don Giovanni, qui voyage de l'une à l'autre et n'appartient plus ni à l'une, ni à l'autre. Dans le livret le Commandeur est censé courir, mais le rythme de son phrasé suggère des pas lourds et une attitude solennelle. Les vers sont plus courts, de cinq syllabes, et les intervalles plus amples; les accents scandés préparent l'entrechoc des armes. Le Battiti (Bats-toi!) avec lequel il défie le séducteur se confond avec le Pentiti (Repens-toi!) de la scène finale. Le combat, d'abord refusé par Don Giovanni, séducteur mais point assassin, se clôt sur la fin du Commandeur racontée sur un mode mineur et par un andante d'extrême douceur et raréfaction, une lente phrase circulaire qui est le long halètement d'un homme qui se meurt. Cette agonie en prise directe est physiquement exprimée par l'évocation du «sein palpitant»: mais si l'agonisant «sent» (sento) son âme s'échapper, le séducteur la «voit» (veggo) partir. Leporello écoute son propre égarement d'homme commun qui refuse tout excès, en marge de ce face à face entre un homme qui meurt et un autre qui semble scruter avec étonnement ce mystère de la mort qui se déroule sous ses yeux. Sa fonction de troisième voix, à la fois dans la dispute entre Donn'Anna et Don Giovanni et dans le duel entre ce dernier et le Commandeur, me semble avoir une fonction de basse continue qui ne fait qu'exalter l'opposition entre protagoniste et antagoniste.

Mozart et Da Ponte touchent, dès cette première scène, au noyau du mythe: la transgression des normes et des institutions par un héros qui n'est que mouvement et pulsion vitale, mais aussi le doute sur l'au-delà d'un libertin "hanté par la mort, flatté par la mort» (Montherlant, 1957, p. 13). C'est ce doute qui conduit le libertin à frapper la société à son cœur, dans le brouillage des dispositifs de contrôle de la mort et dans la circulation normée des femmes comme élément premier du fonctionnement social (Lévi-Strauss, 1949). Une remarque de Jouve (1942) sur la structure musicale de la première scène devient ainsi extrêmement parlante à l'ethnologue. Ce critique souligne comment la phrase circulaire de Don Giovanni (Ah già cade il sciagurato, Affannoso e agonizzante) n'est rien d'autre que la phrase circulaire de Donn'Anna (Come furia disperata Ti saprò perseguitar) reprise dans un temps plus lent et sur un mode mineur. Cela me semble montrer non seulement le lien profond entre ces deux personnages, comme le suggère Jouve, mais aussi l'indissociabilité entre le questionnement sur la mort et l'appropriation irrégulière des femmes ${ }^{3}$. Cette circularité et reprise musicale montre aussi un glissement dans la distribution des rôles: l'antagoniste de Don Giovanni n'est plus le seul Convive de pierre, mais aussi la femme en tant qu'instrument de médiation avec la transcendance ${ }^{4}$. La poursuite musicale de la première scène s'arrête stupéfaite sur le Commandeur mourant, le rythme des péripéties, leur succession fébrile, rappellent de près l'enchaînement de questions que Léonce l'insolent, jeune athéiste et disciple de Machiavel, pose au mort dans une des premières versions $d u$ mythe de Don Juan:

Verumne illud est, quod passim credunt mortales, immortalem spiritum humano claudi corpuscolo, illudque ab anima, totum vitae beneficium accipere? quod si tibi aliquando etiam talis hospes inhabitavit, dic obsecro, postquam eccessit, peritne totus, an superest? et si quidem superestes est, ubi moratur, et quibus locis? salvusne est, an plectitur? num alter orbis aeternitatem habet? (Zehentner, dans Macchia, 1978, p. 113).

Est-ce vrai ce à quoi les hommes croient, qu'un esprit immortel est enfermé dans un petit corps mortel et que ce dernier reçoit de l'âme les bienfaits de la vie? Et si autrefois tu as accueilli en toi un tel hôte, dismoi, s'il te plaît, est-il mort ou a-t-il survécu à la mort? Et si jamais il est encore en vie, où habite-t-il? Dans quel lieu? A-t-il trouvé son salut ou sa damnation? Est-ce que cet autre monde est éternel? (Traduction libre.)

Ce questionnement sur la mort gît au cœur de ce que Magli (1987) appelle la structure interrogative de la musique: la tension entre l'achèvement de la forme et l'infinitude de l'homme, entre un sujet qui s'affirme per se et sa justification transcendante. La palpitation de la musique, le défi de Don Juan me semblent ainsi donner corps à la confrontation toujours renouvelée entre l'Es muss sein de Beethoven (qui d'ailleurs n'aimait pas le Don Giovanni) et le panta rei de Héraclite, entre «la pesanteur, la nécessité et la valeur dans le signe du destin » et "l'insoutenable légèreté de l'être» (Kundera, 1984).

\section{DE LA MORT-ANTAGONISTE À LA MORT-OMBRE}

Des auteurs comme Macchia (1978) ou Straus (1990) indiquent dans le Rake's Progress (La carrière d'un libertin) d'I. Stravinsky et W. H. Auden (1951) une des plus récentes versions du mythe. Chew (1993) souligne d'autre part l'influence de la pensée de la philosophie chrétienne de Kierkegaard sur le livret d'Auden, philosophie qui a sa clé de voûte dans la lecture du Don Giovanni mozartien. Néanmoins il me semble que The Rake's Progress constitue plutôt une forme de Don Juan renversé qui nous permet d'éclairer un nouveau rapport à la mort. Stravinsky décide de composer cet opéra après avoir visité à Chicago une exposition consacrée à Hogarth et en particulier à une série de tableaux datant de 1732-1733 que le peintre anglais avait consacré à la vie d'un jeune homme de la bonne société qui, après avoir touché un héritage, le gaspille au jeu et avec les filles, pour finir ensuite dans un asile d'aliénés. Seule la femme aimée et délaissée restera à ses côtés à l'heure de sa mort, le berçant dans ses bras comme un enfant.

Le premier tableau s'ouvre aussi sur un jardin, celui de Trulove, l'homologue du Commandeur et père d'Anne, la fiancée de Tom Rakewell ${ }^{5}$. Comparé toutefois au dynamisme et à la mobilité affective de la première scène du Don Giovanni mozartien, ce tableau est plutôt une représentation «assise» où le seul mouvement de fuite est celui dans la rêverie ou dans le souvenir, comme le montre bien la rime stream dream dans le duo qui ouvre la scène. Tom est un libertin en pantoufles, un futur mari saisi en train de chanter avec sa fiancée les 
joies de l'amour dans une après-midi de printemps: Anne: How sweet within the budding grove To walk, to love. How sweet beside the pliant stream To lie, to dream. How sweet/ Tom: How sweet beside the pliant stream To lie, to dream. How sweet within the budding grove To walk, to love. How sweet. La douceur languissante renforcée par le timbre des bois, ainsi que par la sonorité et la récursivité du how sweet, ne pourrait pas être plus lointaine de la fuite donjuanesque. Cette inertie dans un hortus clausus qui ne connaît ni chagrin, ni haine, ni dédains, ni regrets est à peine troublée par les remarques de Trulove, un beau-père inquiet de l'avenir de sa fille et par les velléités de Tom qui ne s'imagine pas de vivre toute sa vie en modeste employé. La place réduite que Stravinsky reconnaît avoir accordée dans cette pièce "aux ensembles et à une polyphonie un peu sacrifiée aux accompagnements de style ostinato» (Jacquot, 1982, p. 121), contribue à accentuer cette atmosphère de raréfaction et d'immobilisme.

Le délire d'extension de Don Giovanni, son amour de l'excès, son défi aux limites - physiques, sociales - sont littéralement renversés : le libertin qui vole à la conquête $\mathrm{du}$ monde devient un arriviste, un gueux qui contemple statiquement (Here I stand) la vaste étendue du réel et s'appelle au désir pour l'aider à franchir le pas qui l'amènera à fuir son idylle pastorale pour faire fortune dans la grande ville: The world is so wide: Come, wishes, be horses This beggar shall ride. Le mot wish va déclencher à chaque fois un changement de décor. Dans la première scène, le souhait d'avoir de l'argent va provoquer l'apparition du diable, Nick Shadow, qui lui promet richesse et fortune: figure ambivalente, qui, à la différence de Leporello, est à la fois le maître et le serviteur ${ }^{6}$. Mais cette sémantique du désir est ambivalente. Tom est un héros constamment déchiré entre l'envie et l'action; anti-héroïque, passif, velléitaire, il ne vit pas l'instant qui passe, toujours tiraillé entre la nostalgie du passé et l'attente du futur (all London shall be mine...); sa carrière est une suite de vœux, mais leur réalisation le perd, alors que ses désirs les plus profonds ne sont assouvis qu'en y renonçant. Tout l'opéra me paraît ainsi une vaste fresque de la perte et du renoncement. Tom ne peut être heureux qu'en épousant celle qui lui répugne (la femme barbue Baba la Turque), il obtiendra les faveurs de la maîtresse de bordel après avoir chanté sa nostalgie la plus poignante de l'amour salvateur; sa renonciation à la victoire dans le jeu de cartes avec le diable (I dare not wish; I wish for nothing else) le sauvera.

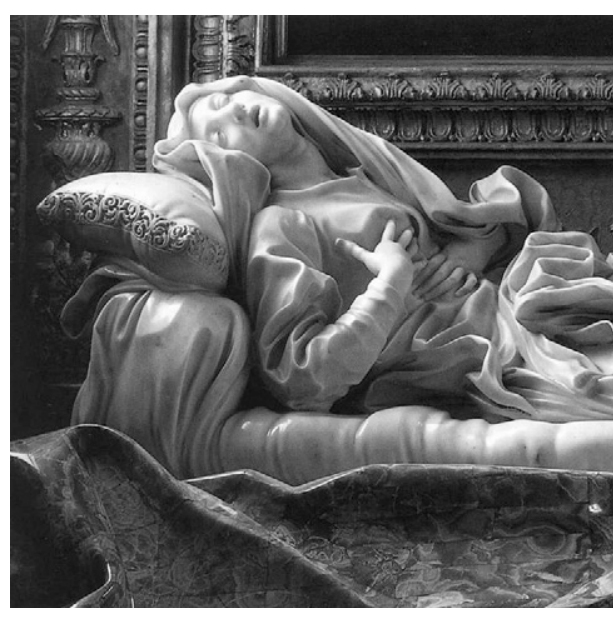

Gian Lorenzo BERNINI, Beata Ludovica Albertoni, 1671-74, Marbre, Cappella Altieri, San Francesco a Ripa, Rome.

L'échec de la logique du désir apparaît dès le premier tableau dans l'histoire racontée par Nick pour justifier l'héritage: elle révèle les péripéties d'un oncle qui, pour l'amour du profit, a quitté son pays, brisé tout lien affectif et familial, renoncé à la consolation d'un dernier baiser sur son lit de mort. Le récit de cet homme malade de nostalgie, sick for his home, sick for a memory of pleasure or of love, n'est qu'une mise en abîme et une préfiguration de la carrière du libertin. Le premier tableau ne se clôt donc pas sur une mort, mais sur une suite d'adieux exprimés sur un ton élégiaque et dominés par l'ombre du pressentiment. Ce sentiment de perte qui domine dès le début relève d'un chavirement fondamental, car The Rake's Progress met en scène la crise des instruments traditionnels de médiation avec l'audelà : aucun Commandeur ne vient parler au nom de Dieu, le ciel est silencieux et l'homme seul face à sa mort, comme Tom devant la tombe vide qui l'attend. La mort n'est pas une antagoniste, mais l'ombre de Rakewell, une partie de lui. L'opposition et la résistance à un paradis fabriqué par les hommes se convertissent en une nostalgie de l'Eden, dont la cavatina teintée de mélancolie du deuxième tableau du premier acte (Love, too frequently betrayed) constitue le sommet musical ${ }^{7}$. Ce renversement investit de la même manière le rapport à la femme, qui n'est plus mû par un insatiable appétit sexuel amenant le héros à briser les liens du mariage, mais par le regret jamais assouvi de l'amour comme lien moral, comme le montrent les références au "véritable amour» qui émaillent l'opéra.

La structure ternaire de l'affrontement, qu'on a vu chez Mozart et Da Ponte ouvrir sur une opposition et une hiérarchie entre sphère mondaine et sphère transcendante, franchies et brouillées par Don Giovanni, laisse la place ici à une succession de tableaux, tant dans la structure générale de l'opéra, que dans celle des scènes singulières - centrées sur une alternance d'airs et de récitatifs - et inspirée de la musique de chambre. Ainsi dans le premier tableau, chaque personnage semble presque s'exprimer de manière indépendante des autres : un avant-goût peut-être de la solitude qui accompagne Tom comme une ombre, son valet Shadow, dans la suite de l'opéra. De la même manière si la carrière du libertin peut être associée à une quête initiatique, dont chacun des souhaits constitue un passage, ce voyage ne se déploie pas dans le temps linéaire et dynamique, mais dans celui fluctuant des rêves et dans le désir inassouvi de la répétition. Le flottement entre tonalité et atonalité me semble évoquer parfois un état quasi somnambulique: les événements se suivent, la cohorte bruyante de la foule modifie son opinion, la ville palpite de l'animation de ses bordels et de ses marchés, mais Tom reste fondamentalement inchangé. L'évocation du cycle du temps (the progress), le renouveau voire la célébration du printemps, conduisent à un retour à l'enfance, comme le montrent la berceuse finale et «les nombreuses citations de nursery rhymes [qui] accusent à la fois l'aspect infantile de la psychologie de Tom et les divertissements irresponsables des Whores et des Roaring Boys » (Felz, 1991, p. 62), des prostituées et des braillards.

La folie dans laquelle Tom sombre par punition ne marque pas, selon moi, "une rédemption» (Jacquot, 1982), mais l'épinglage du libertin dans une condition d'entre-deux. Si Don Giovanni est l'homme sans limites parce qu'il remet en question la démarcation fondamentale entre morts et vivants, Rakewell incarne une figure à liminalité fixée, suspendue entre un état et l'autre, marquée par un passage inachevé. Si la référence au monde classique est centrale dans cet opéra (Jacquot, Vaccaro et Chimènes, 1990), l'asile me paraît toutefois moins proche de l'enfer des anciens, que de cette contrée de l'entre-deux où errent ceux qui n'ont pas de sépulture, un lieu paradoxal d'enfermement et d'errance où vaguent les oubliés des vivants. Le défi de Don Juan est pensable dans un humus culturel qui impose le culte des morts et, par là, leur restitue une présence ontologique dans l'espace social et moral des vivants. La mort s'impose avec la concrétude du marbre blanc, lourd, opaque du Convive de Pierre, véritable alter ego du sujet. Deux siècles plus tard, le séducteur indécent s'éclipse derrière l'indécence de la mort. La carrière de Rakewell exprime l'angoissante solitude de celui qui ne connaît qu'une seule mort, la sienne, et le désarroi d'une société 
qui se fonde sur «la disparition de la mort comme limite et sur le traitement de la mort comme disparition» (Baudry, 1999, p. 40). C'est son ombre, Nick Shadow, qui s'engouffre dans le tombeau vide, avant d'abandonner le libertin à l'absence-présence de la folie, dans le monde d'ombres de Bedlam, où il rejoue dans un printemps éternel the holy rites of love, les "saints rites de l'amour ${ }^{8}$ ", à l'usage d'un aveugle, d'un invalide, d'un homme avec un télescope et de trois vieilles sorcières.

\section{Bibliographie}

ABERT, H. (1985 [1955]). W.A. Mozart, Milan, Il Saggiatore.

BAUDRY, P. (1999). «L'espacement des morts ", Cultures en mouvement, $\mathrm{n}^{\circ} 20$, p. 40-42.

BYRON, G.G. (1982 [1818]). Don Juan, Milan, Mondadori.

CAMUS, A. (1985). Le mythe de Sisyphe, Paris, Gallimard.

CHEW, G. (1993). "Pastoral and neoclassicism: A reinterpretation of Auden's and Stravinsky's Rake's Progress », Cambridge Opera Journal, vol. 5, n 3, p. 239-263.

DE SIMONE, R. (1979). «Il culto », La voce della Campania, $\mathrm{n}^{\circ}$ 18, p. 51-52.

DIASIO, N. (1987). Tempo della morte e tempo della seduzione. Una lettura antropologica del personaggio di Don Giovanni, Tesi di Laurea, Università di Roma La Sapienza.

DUMAS, A. (1863-1864 [1836]). « Don Juan de Marana ou la chute d'un ange», dans Théâtre Complet, Paris, Lévy frères, vol. 3, p. 313-411.

FARINELLI, A. (1946). Don Giovanni, Milan, Bocca.

FELZ, N. (1991). "The Rake's Progress: masque élisabéthain sous un loup vénitien », Revue de musicologie, vol. 77, $\mathrm{n}^{\circ} 1$, p. $59-80$.

GARBOLI, C. (1983). "Come recita Don Giovanni ", dans Studi in onore di Giovanni Macchia, Milan, Mondadori, p. 284-308.

GENDARME DE BEVOTTE, G. (1911). La légende de Don Juan: son évolution dans la littérature des origines au Romantisme, Paris, Hachette.

GOLDONI, C. (1913-1928). "Don Giovanni Tenorio o sia il dissoluto ", dans Opere complete, Venise, Municipio, vol. XXIII, p. 273-360.

GRABBE, C.D. (1968 [1829]). Don Juan und Faust, tr. it. Don Juan e Faust, Milan, Rizzoli.

HOFFMANN, E.T.A. (1969 [1813]). Don Juan, tr. it. «Don Giovanni. Favolosa avventura accaduta a un Viaggiatore entusiasta », dans Romanzi e racconti, Turin, Einaudi.

JACQUOT, J. (1982). «The Rake's Progress et la carrière de Stravinsky", Revue de musicologie, vol. $68, \mathrm{n}^{\text {os }} 1-2$, p. 110-127.
JACQUOT, J., J.-M. VACCARO et M. CHIMÈNES (1990). The Rake's Progress, Paris, Éd. du CNRS.

JOUVE, P.-J. (1942). Le Don Juan de Mozart, Fribourg, Librairie de l'Université.

KIERKEGAARD, S. (1978 [1843]). Enten Eller, tr. it. Enten-Eller [Aut-Aut], Milan, Mondadori, p. 1-3.

KUNDERA, M. (1984). L'insoutenable légèreté de l'être, Paris, Gallimard.

LENAU, N. (1913 [1844]) Don Juan, traduit et introduit par Walter THOMAS, Paris, Montaigne-Aubier.

LÉVI-STRAUSS, C. (1949). Les structures élémentaires de la parenté, Paris, PUF.

LUHMANN, N. (1985 [1982]). Liebe als Passion. Zur Codierung von Intimität, tr. it. Amore come passione, Bari, Laterza.

MACCHIA, G. (1978). Vita, avventure $e$ morte del Don Giovanni, Turin, Einaudi.

MAGLI, I. (1987). La Madonna, Milan, Rizzoli.

MILA, M. (1988). Lettura del Don Giovanni di Mozart, Turin, Einaudi.

MOLIÈRE (1971 [1664]). Don Juan ou le festin de pierre, Paris, Larousse.

MOLINA, T. DE (1984 [1630]) El Burlador de Sevilla, tr. it. «Il seduttore di Siviglia e Convitato di Pietra", dans Teatro, Turin, UTET, p. 209-301.

MONTAIGNE, M. DE (1965). Essais, Paris, Folio, vol. 3.

MONTHERLANT, H. DE (1957). «Don Juan », La table ronde, p. 119

MUSSET, A. DE (2006 [1832]). Namouna, dans Poésies complètes, Paris, Livre de poche classique.

NIOLA, M. (2003). Il purgatorio a Napoli, Rome, Meltemi.

PUŠKIN, A. (1985 [1830]). Kamenyi Gost, tr. it. "Il convitato di pietra», dans Mozarte Salieri e altri microdrammi, Turin, Einaudi, p. 17-54.

RANK, O. (1973 [1932]). Don Juan et le double, Paris, Pavot.

ROSTAND, E. (1921). La dernière nuit de Don Juan, Paris, Fosquelle.

ROUSSET, J. (1978). Le mythe de Don Juan, Paris, Armand Colin.

STRAUS, J. (1990). Remaking the Past: Musical Modernism and the Influence of the Tonal Tradition, Cambridge (Mass.), Cambridge University Press.

ZEHENTNER, P. (1978 [1615]). «Promontorium malae spei impiis periculose navigantibus propositum », dans G. MACCHIA, Vita, avventure e morte del Don Giovanni, Turin, Einaudi, p. 109117.

ZORRILLA, J. (1957 [1844]). Don Juan Tenorio, Milan, Rizzoli.

\section{Discographie}

MOZART, W.A. et L. DA PONTE (1961 [1787]). Don Giovanni, interprété par C. M.
GIULINI (dir.), Philarmonia Chorus and Orchestra, EMI Records.

STRAVINSKY, I. et W.H. AUDEN (1951). The Rake's Progress, interprété par R. CHAILLY (dir.), London Sinfonietta, Decca Digital.

\section{Notes}

1. Voir une version contemporaine de l'histoire du Capitan, invité au banquet nuptial par raillerie par un jeune mafieux, coureur de jupons, qui non seulement refuse de pratiquer les rites liés au culte des âmes du purgatoire dans le cimetière destiné à cet effet, mais le profane en y faisant l'amour avec une de ses conquêtes (De Simone, 1979).

2. La didascalie souligne que Donn'Anna «retient fortement Don Giovanni par le bras », ce qui a donné lieu aux lectures romantiques de la victime amoureuse de son séducteur (voir Hoffmann, 1813).

3. Prenons un contre-exemple musical: Falstaff est impuissant et sa séduction burlesque car il ne remet pas en question les deux formes de maîtrise des aléas de l'existence : la circulation des femmes - et donc le contrôle sur la reproduction - et la domestication sociale de la mort.

4. Les personnages féminins des précédentes versions n'étaient que des pâles comparses. Dans la première scène de l'opéra de Mozart et Da Ponte, par contre, c'est Donn'Anna qui prend la parole en premier et ce changement de rôle a été bien ressenti par les auteurs romantiques (par exemple: Hoffmann, 1813; Grabbe, 1829; Puškin, 1830 ; de Musset, 1832; Dumas père, 1836; Zorrilla, 1844 ; Lenau, 1844) qui en feront le personnage principal, en opérant un déplacement de la sémantique du plaisir à celle de l'amour tout à fait conforme aux nouvelles formes de l'intimité à la fin du XVIII ${ }^{\mathrm{e}}$ siècle (Luhmann, 1982)

5. Dans ce conte moral, tous les personnages ont des noms qui en trahissent le caractère et la fonction: Anne Trulove, le «véritable amour », Tom Rakewell, le libertin, Nick Shadow, le serviteur-diable (Nick étant le nom familier du diable en pays anglo-saxon et Shadow de l'ombre).

6. Voir le récitatif secco qui marque l'entrée en scène du diable: Tom: Tom Rakewell at your service. Nick: Nick Shadow, sir, and at your service.

7. Cette nostalgie se prévaut aussi d'une alternance entre musique tonale et atonale: "When Stravinsky is depicting a harmonious world, a golden age, the traditional elements predominate, as in the Garden. When the world appears bleak and hopeless, as in the Graveyard, the balance is shifted the other way. The motive, because of its inherent dual nature, is able to function in both musical worlds» (Straus, dans Chew, 1993, p. 251).

8. Cette compulsion de répétition rappelle la fin de Don Juan racontée par Rostand (1921): un grand guignol où il joue sans arrêt les vicissitudes du Burlador: le devenir de la musique figé dans les gestes mécaniques d'une marionnette sans cœur, sans corps. 